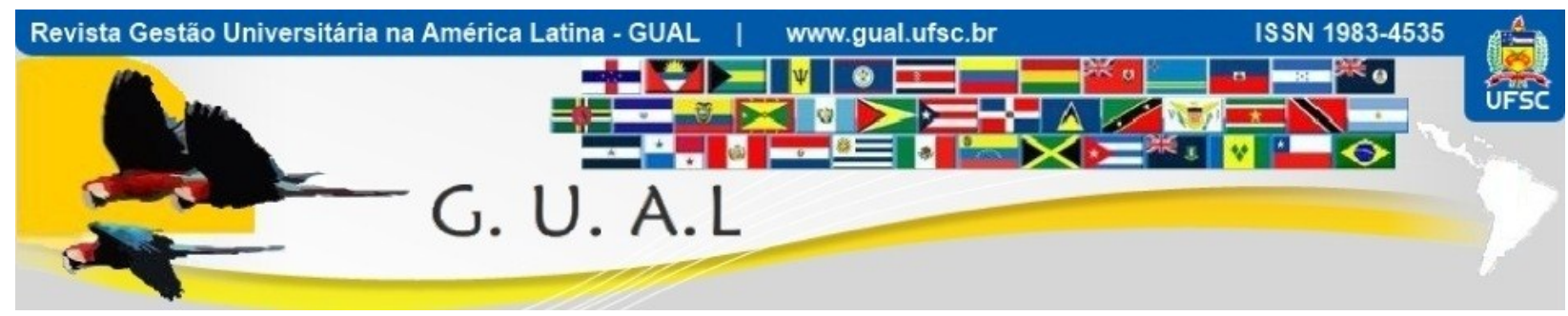

DOI: http://dx.doi.org/10.5007/1983-4535.2013v6n2p160

\title{
UM PANORAMA SOBRE OS ESTUDOS RELACIONADOS À TEMÁTICA EDUCAÇÃO E SUSTENTABILIDADE
}

\section{AN OVERVIEW ON STUDIES RELATED TO EDUCATION AND SUSTAINABILITY}

Adilson Carlos da Rocha, Mestrando

Universidade Federal de Santa Maria - UFSM adilson28@hotmail.com

Caroline Rosseto Camargo, Mestranda Universidade Federal de Santa Maria - UFSM carolinerc@,gmail.com

Jordana Marques Kneipp, Doutoranda Universidade Federal de Santa Maria - UFSM jordana.mk@gmail.com

Lucas Veiga Ávila, Mestrando Universidade Federal de Santa Maria - UFSM admlucasveiga@gmail.com

Lúcia Rejane da Rosa Gama Madruga, Doutora Universidade Federal de Santa Maria - UFSM lucia.rejane@hotmail.com

Recebido em 16/janeiro/2013

Aprovado em 17/abril/2013

Sistema de Avaliação: Double Blind Review

Esta obra está sob uma Licença Creative Commons Atribuição-Uso. 


\title{
RESUMO
}

O estudo tem como objetivo analisar as características das publicações sobre Educação e Sustentabilidade (Education and Sustainability), nas bases de dados Web of Science (WOS) e Scopus, no período de 1991 a 2012, e identificar quais tópicos estão sendo estudados junto a este tema e quais são os mais relevantes (hot topics), realizando uma comparação dos resultados encontrados nas duas bases de dados (WOS e Scopus). O estudo possui abordagem quantitativa tendo em vista que procurou quantificar algumas variáveis referentes à produção científica sobre o tema pesquisado. A busca das publicações sobre Educação e Sustentabilidade no período de 1991 a 2012, encontrou 2.419 publicações na base WOS e 5.124 na Scopus. Comparando-se ambas as bases pesquisadas verificou-se que o número de publicações aumentou de forma expressiva na última década e que os Estados Unidos lidera o ranking quanto ao número de publicações. Percebe-se que em ambas as bases de dados, os autores das publicações mais citadas não estão entre os autores que mais publicaram no período. Constatou-se que as publicações com maior número de citações estão relacionadas à área da saúde, e, as publicações que ocupam $1^{\circ}$ e $2^{\circ}$ lugar no ranking das mais citadas são as mesmas tanto na base WOS quanto na Scopus.

Palavras-chave: Sustentabilidade. Educação. Bibliometria.

\begin{abstract}
The study aims to analyze the characteristics of publications on Education and Sustainability (Education and Sustainability), the databases Web of Science (WOS) and Scopus for the period 1991 to 2012, and identify what topics are being studied at this theme and what are the most relevant (hot topics), making a comparison of the results in two databases (WOS and Scopus). The study has a quantitative approach in order that sought to quantify some variables related to the scientific literature on the topic searched. A search of publications on Education and Sustainability in the period from 1991 to 2012, found 2419 publications based on WOS and Scopus in 5124. Comparing both bases surveyed it was found that the number of publications increased dramatically in the last decade and that the United States leads the ranking as the number of publications. We notice that in both databases, the authors of the most cited publications are not among the authors who published in the period. It was found that the publications with the highest number of citations are related to health, and the publications that occupy 1 st and 2 nd place in the ranking of most frequently cited are the same as in the base WOS Scopus.
\end{abstract}

Keywords: Sustainability. Education. Bibliometrics. 


\section{INTRODUÇÃO}

As discussões relacionadas ao desenvolvimento sustentável do planeta são cada vez mais recorrentes em diferentes contextos e áreas do conhecimento. Em virtude dos inúmeros problemas sociais e ambientais que vêm ocorrendo nas últimas décadas, a fim de garantir condições de sobrevivência para as gerações futuras, são crescentes os movimentos em prol do desenvolvimento sustentável, definido como o "desenvolvimento capaz de suprir as necessidades da geração atual, sem comprometer a capacidade de atender as necessidades das futuras gerações (CMMAD, 1991, p. 9)".

A crise ambiental e social ocorrida no início dos anos de 60 impulsionou a emergência da temática referente ao desenvolvimento sustentável, que passou a ser difundida em meados da década de 1980 e vem sendo debatida por muitos grupos e de diversas maneiras nos mais amplos espaços de diálogo acadêmico ou empresarial (BARBIERI, 2007; BRITO; LOMBARDI, 2007).

Hoff (2008) ressalta que tanto as discussões teóricas quanto as iniciativas científicas giram em torno da busca por soluções para a redução do impacto das atividades humanas sobre o meio ambiente, e, ao mesmo tempo, buscam mecanismos para melhorar a distribuição das riquezas e o bem-estar entre indivíduos e regiões.

O debate referente ao desenvolvimento sustentável somente possui sentido ao buscar subsídios para um processo de mudanças, no qual a exploração de recursos, a direção dos investimentos e a orientação do desenvolvimento tecnológico das organizações e da sociedade tratem os recursos naturais como finitos, considerando que a sua má utilização pode levar a um colapso global (BRITO; LOMBARDI, 2007).

As discussões relacionadas ao desenvolvimento sustentável trazem novas perspectivas para as empresas e para a sociedade. Para Hoff (2008), a sustentabilidade requer um novo paradigma de desenvolvimento, que integre crescimento econômico, preocupação social e proteção ambiental como elementos interdependentes que visam o longo prazo e que se suportam mutuamente.

Nesse panorama, a educação tem sido um elemento fundamental. Zitzke (2002) destaca que a educação possui importante papel para a sustentabilidade, atuando como tradutora do conhecimento técnico e científico para a compreensão de todos os envolvidos com as questões ambientais, podendo induzir ao pensamento crítico e a busca por 
demonstrações ou justificativas, de modo que o indivíduo passe a não aceitar sem analisar, a implantação de qualquer projeto que ofereça riscos sociais ou ambientais.

Diante desse contexto torna-se importante ampliar o conhecimento referente à temática educação e sustentabilidade. O presente estudo tem como objetivo geral analisar as características das publicações sobre educação e sustentabilidade nas bases de dados Web of Science (WOS) e Scopus. Tendo como objetivos específicos: identificar os hot topics relacionados à educação e sustentabilidade, e identificar os principais artigos citados.

Este trabalho está estruturado em quatro seções além desta introdução. A primeira apresenta a contextualização teórica sobre educação e sustentabilidade, a segunda seção trata dos procedimentos metodológicos do estudo. Logo após, apresenta-se a análise e discussão dos resultados. Por fim, apresentam-se as considerações finais da pesquisa.

\section{A EDUCAÇÃO PARA A SUSTENTABILIDADE}

A educação para a sustentabilidade representa um novo paradigma para o ensino e a aprendizagem. O tema implica em uma nova forma de pensar e de aprender, em novos comportamentos pessoais e de tomada de decisão, trazendo consigo novos conhecimentos e habilidades, para o campo da ciência, da pesquisa e para as organizações (NOLET; GILDA, 2010).

Para Gabriel e Morriguchi (2005) a educação é um elemento fundamental para a sustentabilidade, pois é possível formar cidadãos com a capacidade de enfrentar as mudanças que vem ocorrendo em todos os níveis da sociedade. Segundo Tamaio (2000), em virtude dos inúmeros problemas ambientais, vários setores sociais almejam que o processo educativo venha a provocar mudanças e alterar a situação crítica de degradação do meio ambiente.

$\mathrm{Na}$ visão de Rowe (2005), um dos grandes desafios da educação é contribuir na formação de um indivíduo integrante e atuante na sociedade. Nesse contexto, a universidade por meio do ensino, da pesquisa e da extensão, assume um importante papel na preparação de novas gerações com pensamento voltado para as questões relacionadas ao desenvolvimento de uma sociedade mais sustentável, sendo a educação um dos caminhos para realização deste processo (STEPHENS et al, 2008).

De acordo com Dias (2007), a educação prepara os estudantes para desenvolverem uma visão crítica, de forma a criticar e a repensar o modo de ver a sociedade, contribuindo para a internalização do conceito de gestão ambiental. Jacobi (2003) enfatiza que a educação 
assume cada vez mais, uma posição de transformadora de opiniões e atitudes, uma vez que estimula os indivíduos a se tornarem responsáveis por promover o desenvolvimento sustentável no meio em que vivem.

Educar para a sustentabilidade representa um novo desafio no campo educacional, tendo em vista a complexidade e importância deste processo na formação de uma geração mais consciente e engajada com a sustentabilidade do Planeta. Complementando, Dias (2007) coloca que a educação para a sustentabilidade é um processo bastante complexo e não pode ser considerado de forma fragmentada.

Para Gadotti (2008, p. 67) a educação para o desenvolvimento sustentável busca fornecer caminhos a fim de utilizar a educação como meio para construir um futuro mais sustentável e requer ir além da discussão teórica sobre o tema. Na visão do mesmo autor, a educação para o desenvolvimento sustentável propicia a aprendizagem de atitudes, perspectivas e valores que orientam e impulsionam as pessoas a viverem de forma sustentável, tomando consciência dos danos causados pelos seres humanos ao Planeta e estimulando o desenvolvimento de ações a fim de minimizar os problemas socioambientais.

Jacobi (2003) destaca o importante papel do professor como agente impulsionador das transformações em prol de uma educação que assume um compromisso com a formação de valores de sustentabilidade, como parte de um processo coletivo.

No ano de 2002, a Assembleia Geral das Nações Unidas proclamou a Década da Educação das Nações Unidas para o Desenvolvimento Sustentável, com duração de 2005 a 2014. A Organização das Nações Unidas para a Educação, a Ciência e a Cultura (UNESCO) foi escolhida para liderar o processo, que possui como objetivo global integrar os valores inerentes ao desenvolvimento sustentável em todos os aspectos da aprendizagem com o intuito de fomentar mudanças de comportamento que permitam criar uma sociedade sustentável e mais justa (UNESCO, 2005).

O Plano de implementação da Década da Educação das Nações Unidas para o Desenvolvimento Sustentável inclui importantes dimensões, tais como: direitos humanos, paz e segurança humana, igualdade de gênero, diversidade cultural e compreensão intercultural, saúde, governabilidade, recursos naturais, mudanças climáticas, desenvolvimento rural, urbanização sustentável, prevenção e atenuação de desastres naturais, redução da pobreza, responsabilidade e deveres das empresas e economia de mercado (UNESCO, 2005). 
Segundo Gadotti (2008) várias regiões já definiram as suas estratégias de implementação da Década, dentre elas: a Europa, a região da Ásia-Pacífico, a América Latina e o Caribe. Na visão do autor, na estratégia da Europa definida em 2005, destacam-se os objetivos de formação dos educadores para a inclusão do desenvolvimento sustentável na sua atividade docente e a garantia de acesso aos instrumentos e materiais necessários à educação para o desenvolvimento sustentável.

A América Latina definiu sua estratégia regional em 2006, na qual se destacam: a articulação de esforços convergentes, a integração e harmonização da política educativa em cada país com a educação para o desenvolvimento sustentável, o fortalecimento de marcos normativos e políticas públicas para o seu incremento, a comunicação e a informação do conceito de sustentabilidade e o fortalecimento da cooperação e da associação estratégica entre os diferentes setores e agentes do âmbito público, privado e da sociedade civil organizada (GADOTTI, 2008).

No Brasil, a relação entre Educação e Sustentabilidade está presente como exigência e determinação desde a Constituição Federal Brasileira de 1988, que no artigo 214, estabelece o sistema nacional de educação em regime de colaboração e apresenta definições de diretrizes, objetivos, metas e estratégias de implementação procurando assegurar a manutenção e o desenvolvimento do ensino em seus diversos níveis (CFB, 1988, p. 127-28).

Além da CFB, o Conselho Nacional de Educação - CNE retoma esta questão no artigo 15 da Lei Federal $n^{\circ}$ 9.795, que dispõe sobre a educação ambiental, instituindo a Política Nacional de Educação Ambiental e dá outras providências: Por meio do capítulo I (artigos $1^{\circ}$, $2^{\circ}$ e $3^{\circ}$ ) estabelece que a educação ambiental é componente essencial e permanente da educação nacional, devendo estar presente, de forma articulada, em todos os níveis e modalidades do processo educativo, em caráter formal e não formal (LEI FEDERAL 9.795, 1999).

A partir deste movimento em prol do desenvolvimento sustentável na educação brasileira, em especial na grade curricular das disciplinas de cursos de graduação, foi proposta a inserção da sustentabilidade na formação do discente. Por meio desta proposta, o Presidente do CNE em conformidade com o disposto na Lei ${ }^{\circ} 9.394$ do CNE, de 20 de dezembro de 1996, e no parecer $\mathrm{n}^{\mathrm{o}}$ 14/2012, publicado no DOU de 15 de junho de 2012, aprovou as Diretrizes Curriculares Nacionais para Educação Ambiental (DOU, 2012). A presente resolução estabelece as Diretrizes Curriculares Nacionais para a Educação Ambiental a serem 
observadas pelos sistemas de ensino e suas instituições de Educação Básica e de Educação Superior (MEC, 2012).

Verifica-se, que no Brasil há mais de duas décadas o processo de educação com vistas para a sustentabilidade encontra-se em andamento. Após a conferência Rio +20 , o MEC, por meio da resolução, propôs novos mecanismos para inserção da sustentabilidade no ensino.

Segundo Engelman, Fracasso e Tometich (2012) a educação torna-se um aliado importante na difusão da sustentabilidade, pois o ensino pode preparar profissionais que sejam capazes de usar seus conhecimentos para promover ações que atendam as necessidades sociais e ambientais nos diferentes ambientes por onde circulam. $\mathrm{O}$ aluno deve fazer parte de um processo educacional holístico, em que sejam desenvolvidas habilidades em prol da sustentabilidade, por meio de uma pedagogia criativa, para que o discente aprenda a tomar decisões, realizar ações a partir de critérios, normativas, regras, que não prejudiquem o desenvolvimento sustentável.

\section{MÉTODO}

\subsection{Tipo de estudo}

O presente estudo foi desenvolvido a partir de uma pesquisa bibliométrica, objetivando ampliar o conhecimento referente às publicações relacionadas aos temas Educação e Sustentabilidade (Education and Sustainability), nas bases de dados WOS e Scopus. Segundo Silva (2004) a bibliometria possui como objetivo analisar a atividade científica ou técnica por meio do estudo quantitativo das publicações. Complementando esta ideia, Rostaing (1997) coloca que o estudo bibliométrico consiste na aplicação dos métodos estatísticos ou matemáticos sobre o conjunto de referências bibliográficas. Para Macedo, Casa Nova e Almeida (2007) a bibliometria ajuda a conhecer o estágio em que uma pesquisa em determinada área se encontra.

$\mathrm{O}$ estudo possui abordagem quantitativa tendo em vista que procurou quantificar algumas variáveis referentes à produção científica sobre Educação e Sustentabilidade (Education and Sustainability).

\subsection{Definição da amostra}

Os dados para realização desta pesquisa foram coletados nas bases de dados WOS do Institute for Scientific Information (ISI) e Scopus da Elsevier. 
A WOS consiste em uma base multidisciplinar que indexa somente os periódicos mais citados em suas respectivas áreas. É também um índice de citações na web, que além de possibilitar a identificação das citações recebidas, referências utilizadas e registros relacionados, permite analisar a produção científica com cálculo de índices bibliométricos e o percentual de auto-citações, assim como a criação de rankings por inúmeros parâmetros. Possui atualmente cerca de 12.000 periódicos indexados (CAPES, 2012).

As referências de todos os itens indexados são extraídas e a interface das referências citadas demonstram todas as citações de trabalhos às obras de um autor, independentemente dos itens citados serem indexados pela WOS ou não (BAR-ILAN, 2008).

A Scopus consiste em uma base de dados das áreas de Ciências Biológicas, Ciências da Saúde, Ciências Físicas e Ciências Sociais. Indexa mais de 15 mil periódicos, cerca de 265 milhões páginas da Internet, 18 milhões de patentes, além de outros documentos. Dispõe de funcionalidades de apoio à análise de resultados - bibliometria (CAPES, 2012).

A coleta dos dados foi realizada a partir dos mecanismos de busca da WOS e da Scopus, com as palavras-chave: Educação e Sustentabilidade (Education and Sustainability), delimitando a coleta de dados para o período de 1991 a 2012.

\subsection{Modelo conceitual}

Para proceder à análise bibliométrica o estudo buscou identificar as variáveis dispostas no Quadro 1, de modo que a partir das funcionalidades de cada uma das bases de dados foram delimitadas as características a serem investigadas na WOS e na Scopus.

\begin{tabular}{|c|c|c|}
\hline Características gerais das publicações & $\begin{array}{c}\text { WOS } \\
1991-2012\end{array}$ & $\begin{array}{c}\text { SCOPUS } \\
1991-2012\end{array}$ \\
\hline Total de publicações & $\mathrm{X}$ & $\mathrm{X}$ \\
\hline Áreas temáticas & $\mathrm{X}$ & $\mathrm{X}$ \\
\hline Tipos de documentos & $\mathrm{X}$ & $\mathrm{X}$ \\
\hline Ano das publicações & $\mathrm{X}$ & $\mathrm{X}$ \\
\hline Autores & $\mathrm{X}$ & $\mathrm{X}$ \\
\hline Título das fontes & $X$ & $\mathrm{X}$ \\
\hline Instituições & $\mathrm{X}$ & $\mathrm{X}$ \\
\hline Países & $X$ & $\mathrm{X}$ \\
\hline Idiomas & $\mathrm{X}$ & $\mathrm{X}$ \\
\hline Índice $\mathrm{h}-\mathrm{b}$ & $\mathrm{X}$ & \\
\hline Índice $\mathrm{m}$ & $\mathrm{X}$ & \\
\hline Autores versus citacões & $\mathrm{X}$ & $\mathrm{X}$ \\
\hline
\end{tabular}

Quadro 1 Modelo Conceitual para análise bibliométrica 
$\mathrm{Na}$ base de dados WOS foram analisados os índices $\mathrm{h}-\mathrm{b}$ e $\mathrm{m}$ para posteriormente realizar-se a análise dos hot topics. O h-index (índice-h) foi proposto por Hirsch (2005) na pesquisa denominada "An index to quantify an individual's scientific research output" como forma de caracterizar a produção científica de um pesquisador. Hirsch (2005) parte do princípio de que, a quantificação do impacto e a relevância da produção científica individual são muitas vezes necessárias para a avaliação de pesquisadores e comparação de propósitos de pesquisa.

Posteriormente, Banks (2006) propôs o índice h-b uma extensão do h-index, que é obtido por meio do número de citações de um tópico ou combinação em determinado período, listados em ordem decrescente de citações. $\mathrm{O}$ índice h-b é encontrado em publicações que tenham obtido um número de citações igual ou maior à sua posição no ranking. Banks (2006) também explica o cálculo do índice m, o qual é obtido pela divisão do índice "h-b" pelo período de anos que se deseja obter informações (n). Para a análise dos índices h-b e m, foram utilizadas as definições de Banks (2006) evidenciadas no Quadro 2.

\begin{tabular}{|c|l|}
\hline Índice $\boldsymbol{m}$ & \multicolumn{1}{c|}{ Tópico/combinação } \\
\hline $0<\mathrm{m} \leq 0,5$ & $\begin{array}{l}\text { Pode ser de interesse para pesquisadores em um campo específico de pesquisa, o } \\
\text { qual engloba uma comunidade pequena; }\end{array}$ \\
\hline $0,5<\mathrm{m} \leq 2$ & $\begin{array}{l}\text { Provavelmente pode se tornar um "hot topic" como área de pesquisa, no qual a } \\
\text { comunidade é muito grande ou o tópico/combinação apresenta características } \\
\text { muito interessantes; }\end{array}$ \\
\hline $\mathrm{m} \geq 2$ & $\begin{array}{l}\text { É considerado um "hot topic", tópico exclusivo com alcance não apenas na sua } \\
\text { própria área de pesquisa e é provável que tenha efeitos de aplicação ou } \\
\text { características únicas. }\end{array}$ \\
\hline
\end{tabular}

Quadro 2 Definições para classificação de hot topics.

Fonte: Banks (2006).

A partir das definições de Banks (2006) neste estudo serão considerados hot topics as combinações com índice $m \geq 2$.

\subsection{Etapas para a coleta dos dados}

A realização da pesquisa dividiu-se em seis etapas. Inicialmente digitaram-se as palavras: Educação e Sustentabilidade (Education and Sustainability) como tópico no campo de pesquisa das bases WOS e Scopus, delimitando-se o período de 1991 a 2012. Ressalte-se que as duas palavras-chave utilizadas no estudo, foram pesquisadas conjuntamente, na forma de uma expressão. 


\section{UM PANORAMA SOBRE OS ESTUDOS RELACIONADOS À TEMÁTICA EDUCAÇÃO E \\ DOI: http://dx.doi.org/10.5007/1983-4535.2013v6n2p160}

A seguir, nas bases WOS e Scopus foram levantadas as informações: áreas temáticas, tipos de documentos, ano das publicações, autores, título das fontes, instituições, agências financiadoras, países, idiomas e autores versus citações. Destaca-se que o índice h-b e o índice $\mathrm{m}$ para posterior a definição dos hot topics, foram pesquisados somente na base WOS.

$\mathrm{Na}$ segunda etapa foram identificados os tópicos a serem combinados com os tópicos Educação e Sustentabilidade (Education and Sustainability). A partir de uma breve análise das publicações encontradas na primeira etapa foram enumerados 20 tópicos a serem combinados com a expressão Education and Sustainability. Para a seleção dos tópicos utilizou-se como principal critério a relação com o termo pesquisado.

$\mathrm{Na}$ terceira etapa, ocorreu a segunda busca ao sistema, combinando cada um dos tópicos relacionados com o termo Education and Sustainability no período de 22 anos (1991 a 2012). Em seguida, na quarta etapa, foram identificados os "hot topics" por meio do cálculo do índice h-b e m. Na quinta etapa realizou-se uma comparação entre as publicações mais citadas e os autores que mais publicaram no mesmo período. O Quadro 3 evidencia as etapas da pesquisa.

\begin{tabular}{|l|l|}
\hline $\begin{array}{c}\text { Etapas da } \\
\text { pesquisa }\end{array}$ & \multicolumn{1}{c|}{ Descrição } \\
\hline Primeira & $\begin{array}{l}\text { Pesquisa do tópico “Education and Sustainability” na WOS e Scopus; } \\
\text { Análise das características das publicações; }\end{array}$ \\
\hline Segunda & $\begin{array}{l}\text { Identificação dos tópicos relacionados à área de Educação e sustentabilidade; } \\
\text { Foram selecionados 20 tópicos - WOS e Scopus; }\end{array}$ \\
\hline Terceira & Combinação na WOS com os 20 tópicos relacionados; \\
\hline Quarta & Cálculo índice h-b e m e identificar os Hot topics na WOS; \\
\hline Quinta & $\begin{array}{l}\text { Relação entre autores com mais publicações e publicações mais citadas na WOS e } \\
\text { Scopus; }\end{array}$ \\
\hline Sexta & Análise dos dados \\
\hline
\end{tabular}

Quadro 3 Etapas da pesquisa

Desse modo, de acordo com as etapas expostas no Quadro 3 foi realizada a análise bibliométrica do referido estudo, apresentada a seguir.

\section{ANÁLISE E DISCUSSÃO DOS RESULTADOS}

Os resultados da pesquisa evidenciaram as principais características da produção científica relacionada a expressão Education and Sustainability (Educação e Sustentabilidade). Primeiramente foi realizada a pesquisa com o termo na base WOS, no período de 1991 a 2012, sendo encontradas 2.419 publicações. Em segundo momento, 
realizou-se a pesquisa na base de dados da Scopus, sendo encontradas no mesmo período 5.124 publicações, após realizou-se a comparação entre o número de publicações por autor e o número de citações.

\subsection{Características gerais das publicações sobre Education and Sustainability na WOS e Scopus}

A seguir estão apresentadas as características gerais das publicações relacionadas ao tema de acordo com as seguintes categorias: áreas temáticas, tipo de documentos, ano das publicações, autores, título das fontes, instituições, países e idiomas.

\subsection{1 Áreas temáticas das publicações}

O Quadro 4 apresenta as dez principais áreas temáticas relacionadas ao tema de acordo com o número de publicações.

\begin{tabular}{|l|c|l|c|}
\hline \multicolumn{1}{|c|}{ Base WOS Áreas temáticas } & $\begin{array}{c}\mathbf{N}^{\mathbf{0}} \\
\text { publicações }\end{array}$ & \multicolumn{1}{c|}{ Áreas temáticas } & $\begin{array}{c}\mathbf{N}^{\mathbf{0}} \\
\text { publicações }\end{array}$ \\
\hline $\begin{array}{l}\text { Education Research } \\
\text { (Pesquisa Educacional) }\end{array}$ & 706 & $\begin{array}{l}\text { Social Sciences (Ciências } \\
\text { Sociais) }\end{array}$ & 1979 \\
\hline $\begin{array}{l}\text { Environmental Sciences Ecology } \\
\text { (Ciência Ambiental e Ecologia) }\end{array}$ & 534 & Medicine (Medicina) & 1049 \\
\hline Engineering (Engenharia) & 398 & Engineering (Engenharia) & 1037 \\
\hline Business Economics (Economia) & 183 & $\begin{array}{l}\text { Environmental Science } \\
\text { (Ciência Ambiental) }\end{array}$ & 1018 \\
\hline $\begin{array}{l}\text { Public Environmental Health } \\
\text { (Saúde Ambiental Pública) }\end{array}$ & 172 & $\begin{array}{l}\text { Business, Management and } \\
\text { Accounting } \\
\text { (Negócios, Gestão e } \\
\text { Contabilidade) }\end{array}$ & 390 \\
\hline Agriculture (Agricultura) & 137 & Energy (Energia) & 359 \\
\hline $\begin{array}{l}\text { Health Care Sciences Services } \\
\text { (Ciências e Serviços da Saúde) }\end{array}$ & 130 & $\begin{array}{l}\text { Agricultural and Biological } \\
\text { Sciences } \\
\text { (Agricultura e Ciências } \\
\text { Biológicas) }\end{array}$ & 230 \\
\hline $\begin{array}{l}\text { Computer Science } \\
\text { (Ciência da Computação) }\end{array}$ & 87 & $\begin{array}{l}\text { Computer Science } \\
\text { (Ciência da Computação) }\end{array}$ & \begin{tabular}{l} 
Nursing (Enfermagem) \\
\hline $\begin{array}{l}\text { Public Administration } \\
\text { (Administração Pública) }\end{array}$
\end{tabular} \\
\hline $\begin{array}{l}\text { Social Sciences } \\
\text { (Ciências Sociais) }\end{array}$ & 100 & $\begin{array}{l}\text { Earth and Planetary Sciences } \\
\text { (Ciências Planetárias e da Terra) }\end{array}$ & 193 \\
\hline
\end{tabular}

Quadro 4 Áreas temáticas no estudo sobre sustainability and education Fonte: WOS e Scopus (Dez./2012) 
A diversidade de áreas temáticas relacionadas com a temática Education and Sustainability, pode ser observada no Quadro 4, outro fator que pode ser destacado é a diferença entre os resultados encontrados nas duas bases de dados. Enquanto a "Pesquisa para Educação" ocupa o primeiro lugar em número de publicações na WOS, na base Scopus o primeiro lugar é ocupado pelas "Ciências Sociais", área que está em décimo lugar na base WOS. Cabe destacar também que a base Scopus abrange um número maior de publicações quando comparado com a WOS.

\subsubsection{Tipos de documentos}

O Quadro 5 apresenta os tipos de documentos referentes as publicações encontradas na WOS e Scopus.

\begin{tabular}{|l|c|c|l|c|c|}
\hline \multicolumn{4}{|c|}{ Base WOS } & \multicolumn{3}{c|}{ Base Scopus } \\
\hline Tipos de publicação & Frequência & $\mathbf{\%}$ & Tipos de publicação & Frequência & $\%$ \\
\hline Artigos & 1744 & 71.94 & Artigo & 3343 & 65.24 \\
\hline Papers & 610 & 25.59 & Papers & 1064 & 20.77 \\
\hline Resenhas & 104 & 4.31 & Resenhas & 556 & 10.85 \\
\hline Material Editorial & 72 & 3.06 & Breve levantamento & 56 & 1.09 \\
\hline Resenha de Livro & 21 & 0.77 & Nota & 37 & 0.72 \\
\hline Resumo de Reunião & 07 & 0.30 & Editorial & 36 & 0.70 \\
\hline Capitulo de Livro & 03 & 0.12 & Indefinido & 20 & 0.39 \\
\hline Carta & 03 & 0.12 & Carta & 07 & 0.14 \\
\hline \multirow{3}{*}{ Item Bibliográfico } & \multirow{2}{*}{02} & 0.08 & Livro & 03 & 0.06 \\
\cline { 4 - 6 } & & & Relatório & 01 & 0.02 \\
\cline { 4 - 6 } & $2566^{*}$ & $100 \%$ & Errata & 5124 & 0.02 \\
\hline Total & & & & 01 & $100 \%$ \\
\hline
\end{tabular}

Quadro 5 Classificação das publicações quanto ao tipo.

* As publicações foram classificadas em mais de um tipo, desse modo o total é superior ao número total de publicações.

Fonte: WOS e Scopus (Dez/2012)

Em ambas as bases de dados a maioria das publicações encontradas são artigos, correspondendo a $72 \%$ das publicações na WOS e $65 \%$ na Scopus, seguido de papers (mais de $20 \%$ em cada base), evidenciando o caráter científico das mesmas.

\subsubsection{Publicações por ano}

A Figura 2 apresenta a quantidade de artigos publicados por ano relacionados ao tema Education and Sustainability na WOS. 


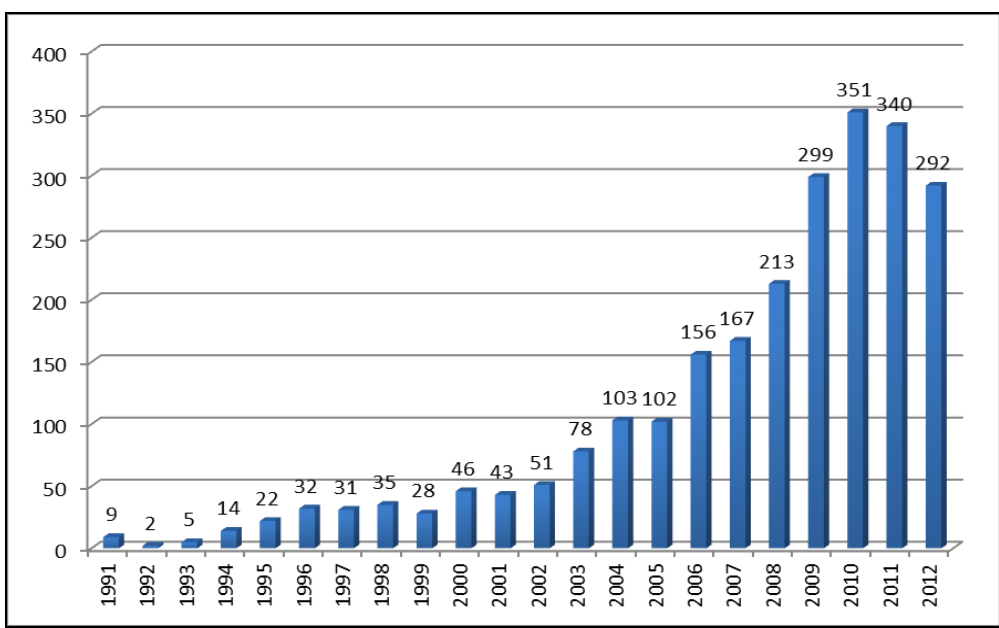

Figura 2 Publicações por ano

Fonte: Base WOS (Dez./2012)

No período de 1991 a 2012 na base de dados da WOS realizando a pesquisa com a expressão Education and Sustainability foram encontrados 2.419 publicações

A Figura 3 apresenta a quantidade de artigos publicados por ano relacionado ao tema Education and Sustainability na base Scopus.

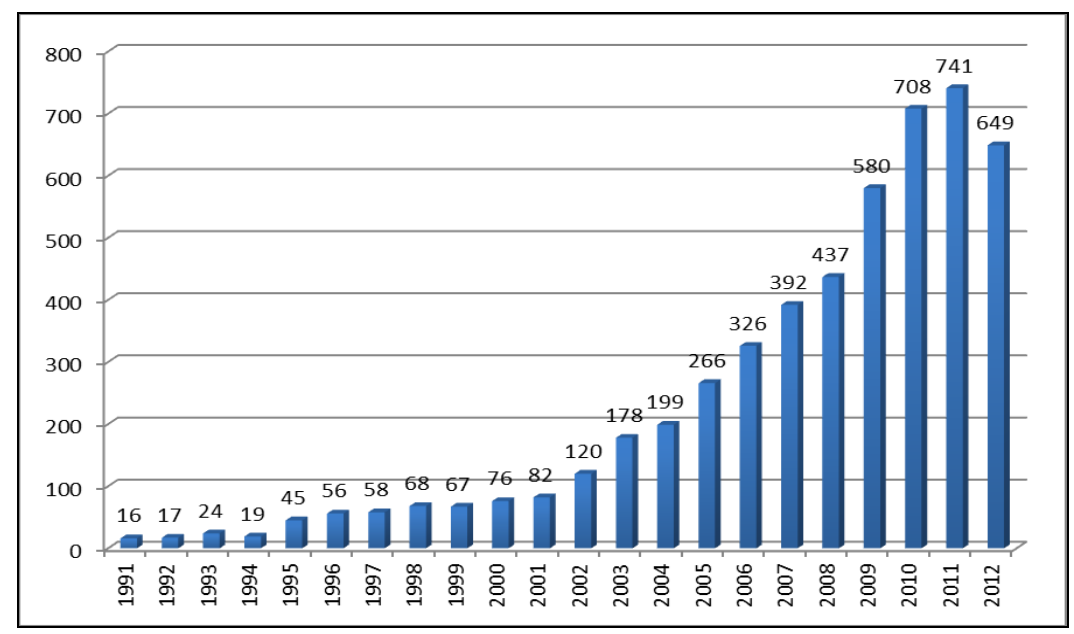

Figura 3 Publicações por ano

Fonte: Scopus (Dez./2012)

No período de 1991 a 2012 na base de dados da Scopus realizando a pesquisa com o tópico Education and Sustainability foram encontradas 5.124 publicações. Comparando o número de publicações no período de 1991 a 2012 nas duas bases pesquisadas, evidencia-se que ano a ano a quantidade de publicações relacionadas ao tema é maior na Scopus. 
É evidente o crescimento no número de publicações nos últimos 10 anos em ambas as bases de dados. Tal fato demonstra a emergência da temática Educação e Sustentabilidade no campo da pesquisa.

\subsubsection{Principais autores}

Os autores que mais publicaram sobre a temática Educação e Sustentabilidade, nas duas bases analisadas, desconsiderando as publicações não assinadas, estão apresentadas no Quadro 6. Foram selecionados os 10 primeiros.

\begin{tabular}{|l|c|l|c|}
\hline \multicolumn{1}{|c|}{ Butor } & \multicolumn{1}{c|}{ Base Scopus } \\
\hline Huisingh, D. & $\begin{array}{c}\text { Artigos } \\
\text { publicados }\end{array}$ & \multicolumn{1}{c|}{ Autor } & $\begin{array}{c}\text { Artigos } \\
\text { publicados }\end{array}$ \\
\hline Barth, M. & 8 & Huisingh, D. & 10 \\
\hline Booth, C. A. & 6 & Mihelcic, J.R. & 10 \\
\hline Fullen, M. A. & 6 & Ferrer-Balas, D. & 9 \\
\hline Scott, W. & 6 & Allenby, B. & 8 \\
\hline Davies, K. & 6 & Barth, M. & 7 \\
\hline Diehl, J. C. & 5 & Davidson, C.I. & 7 \\
\hline Ferrer-Balas, D. & 5 & Haigh, M. & 7 \\
\hline Marinova, D. & 5 & Sutherland, J.W. & 7 \\
\hline Mihelcic, J. R. & 5 & Vanasupa, L. & 7 \\
\hline
\end{tabular}

Quadro 6 Quantidade de artigos publicados por autor Fonte: WOS e Scopus (Dez./2012)

Observou-se uma multiplicidade e diversidade quanto à autoria dos trabalhos, já que uma pequena parcela desses autores publicou um número elevado de artigos sobre a temática. O autor Donald Huisingh lidera o número de publicações nas duas bases de dados, na WOS possui 08 publicações e na Scopus com 10 publicações. Huisingh atualmente é cientista sênior em Desenvolvimento Sustentável da Center Conference localizada nos Estados Unidos da América - EUA. Possui Ph.D. em Bioquímica e Fitopatologia pela Universidade de Wisconsin, BS (EUA); Especialização em Ciência Genética, Biologia e Química pela Universidade de Minnesota (EUA).

$\mathrm{Na}$ base Scopus o autor James R. Mihelcic encontra-se com 10 publicações. James atualmente é Engenheiro Civil e Ambiental, Professor do Estado da Flórida Classe 21st da Century Scholar Mundial localizado nos EUA. Possui Ph. D. em Engenharia Civil, pela 
Universidade Carnegie Mellon, MS Engenharia Civil pela Universidade de Carnegie Mellone, Bacharelado em Engenharia Ambiental pela Universidade Estadual da Pensilvânia.

Além dos autores mencionados, outros autores possuem importantes estudos. Na WOS destacam-se Adam Booth e Mike Fullen com 6 publicações. Já na base Scopus evidenciam-se estudos de Ferrer-Balas e Braden Allenby com 9 e 8 trabalhos publicados, respectivamente.

\subsubsection{Títulos das fontes}

O Quadro 7 apresenta as principais fontes de publicações relacionadas a temática Education and Sustainability.

\begin{tabular}{|l|c|l|c|}
\hline \multicolumn{2}{|c|}{ Bítulo da fonte } & Artigos & \multicolumn{2}{c|}{ Título da fonte } & Artigos \\
\hline Journal of Cleaner Production & 61 & $\begin{array}{l}\text { ASEE Annual Conference and } \\
\text { Exposition Conference Proceedings }\end{array}$ & 247 \\
\hline Environmental Education Research & 58 & $\begin{array}{l}\text { International Journal of } \\
\text { Sustainability in Higher Education }\end{array}$ & 131 \\
\hline $\begin{array}{l}\text { International Journal of sustainability } \\
\text { in Higher Education }\end{array}$ & 53 & Journal of Cleaner Production & 88 \\
\hline $\begin{array}{l}\text { International Journal of Engineering } \\
\text { Education }\end{array}$ & 40 & Sustainability & 67 \\
\hline $\begin{array}{l}\text { WIT Transactions on Ecology and the } \\
\text { Environment }\end{array}$ & 32 & $\begin{array}{l}\text { WIT Transactions on Ecology and } \\
\text { the Environment }\end{array}$ & 41 \\
\hline $\begin{array}{l}\text { International Journal of Educational } \\
\text { Development }\end{array}$ & 23 & $\begin{array}{l}\text { ASEE Annual Conference } \\
\text { Proceedings }\end{array}$ & 37 \\
\hline $\begin{array}{l}\text { Journal of Professional Issues in } \\
\text { Engineering Education and Practice }\end{array}$ & 23 & $\begin{array}{l}\text { International Journal of Engineering } \\
\text { Education }\end{array}$ & 37 \\
\hline $\begin{array}{l}\text { Journal of Geography in Higher } \\
\text { Education }\end{array}$ & 20 & $\begin{array}{l}\text { Proceedings Frontiers in Education } \\
\text { Conference Fie }\end{array}$ & 34 \\
\hline $\begin{array}{l}\text { NATO Science for Peace and Security } \\
\text { series C Environmental Security }\end{array}$ & 18 & Environmental Education Research & 34 \\
\hline $\begin{array}{l}\text { International Journal of Sustainable } \\
\text { Development and World Ecology }\end{array}$ & 17 & $\begin{array}{l}\text { Journal of Professional Issues in } \\
\text { Engineering Education and Practice }\end{array}$ & 28 \\
\hline
\end{tabular}

Quadro 7 Principais fontes

Fonte: WOS e Scopus (Dez/2012)

$\mathrm{Na}$ base WOS as publicações encontradas estão em sua maioria nos seguintes periódicos: Journal of Cleaner Production; Environmental Education Research International; Journal of Sustainability in Higher Education; International Journal of Engineering Education e WIT Transactions on Ecology and the Environment.

$\mathrm{Na}$ base de dados da Scopus evidenciou-se que as publicações pertencem em sua maioria as seguintes fontes: ASSE Annual Conference and Exposition Conference 
Proceedings; International Journal of Sustainability in Higher Education; Journal of Cleaner Production; Sustainability e Wit Transactions on Ecology and the Environment.

Entre os periódicos com maior número de publicações na WOS, destacou-se o periódico que trata especificamente de estudos relacionados à a sustentabilidade, o Journal of Cleaner Production, seguido pelos periódicos com foco nas duas áreas, Education Research Internationa e Journal of Sustainability in Higher Education. Na base de dados da Scopus a primeira colocação ficou com a ASSE Annual Conference and Exposition Conference Proceedings, seguida do International Journal of Sustainability in Higher Education.

\subsubsection{Principais Instituições}

As instituições que mais publicaram trabalhos relacionados ao tema Education and Sustainability estão em destaque no Quadro 8.

\begin{tabular}{|l|c|l|c|}
\hline \multicolumn{2}{|c|}{ Base WOS } & \multicolumn{2}{c|}{ Base - Scopus } \\
\hline Instituição & $\begin{array}{c}\mathbf{N}^{\mathbf{0}} \\
\text { Artigos }\end{array}$ & \multicolumn{1}{c|}{ Instituição } & $\begin{array}{c}\mathbf{N}^{\mathbf{0}} \\
\text { Artigos }\end{array}$ \\
\hline Monash University & 28 & $\begin{array}{l}\text { Virginia Polytechnic Institute and } \\
\text { State University }\end{array}$ & 44 \\
\hline University North Carolina & 21 & The University of British Columbia & 36 \\
\hline University Toronto & 21 & University of Sydney & 34 \\
\hline Arizona State University & 19 & Monash University & 32 \\
\hline Delft University Technol & 19 & Pennsylvania State University & 31 \\
\hline Pennsylvania State University & 19 & University of Queensland & 30 \\
\hline University British Columbia & 19 & Michigan State University & 30 \\
\hline Queensland University Technology & 18 & Queensland University of Technology & 28 \\
\hline University Michigan & 18 & $\begin{array}{l}\text { Royal Melbourne Institute of } \\
\text { Technology University }\end{array}$ & 28 \\
\hline University California Los Angeles & 17 & University of Toronto & 28 \\
\hline
\end{tabular}

Quadro 8 Principais instituições.

Fonte: WOS e Scopus (Dez/2012).

As instituições que mais se destacaram na WOS, foram as seguintes: Monash University (Austrália); University North Carolina (EUA); Pennsylvania State University (EUA); University British Columbia (Canadá); e University Toronto (Canadá). E, na Scopus as seguintes universidades: Virginia Polytechnic Institute and State University (EUA); The University of British Columbia (Canadá); University of Sydney (Austrália); Monash University (Austrália); Pennsylvania State University (EUA). 


\subsubsection{Idiomas e países}

O idioma inglês se sobressai nas duas bases, com $2.332(96,4 \%)$ na base WOS e com $4.954(96,68 \%)$ na base Scopus. O Quadro 9 apresenta os principais países que possuem publicações relacionadas ao tema pesquisado.

\begin{tabular}{|l|c|l|c|}
\hline \multicolumn{2}{|c|}{ Base WOS Países } & Publicações & \multicolumn{2}{c|}{ Pase Scopus } \\
\hline Estados Unidos da América & 839 & Estados Unidos da América & Publicações \\
\hline Inglaterra & 244 & Reino Unido & 1714 \\
\hline Austrália & 242 & Austrália & 494 \\
\hline Canadá & 173 & Canadá & 386 \\
\hline Alemanha & 100 & Alemanha & 173 \\
\hline Holanda & 78 & Holanda & 117 \\
\hline Espanha & 66 & Espanha & 108 \\
\hline China & 62 & China & 106 \\
\hline África do Sul & 46 & Brasil & 89 \\
\hline Escócia & 43 & África do Sul & 81 \\
\hline Brasil & 42 & Índia & 75 \\
\hline
\end{tabular}

Quadro 9 Principais países.

Fonte: WOS e Scopus (Dez/2012).

Os Estados Unidos lideram o ranking de publicações nas duas bases de dados. $\mathrm{Na}$ WOS a Inglaterra é o segundo pais que mais publica, seguida por Austrália, Canadá e Alemanha. Na Scopus, o Reino Unido é o segundo que mais publica, seguido por Austrália, Canadá e Alemanha.

Dentre os países apresentados no Quadro 9, destaca-se que pertencem ao continente Europeu, cinco países na WOS e quatro na Scopus. Este resultado demonstra a importância dada a temática por esta região, que segundo Gadotti (2008) já definiu as suas estratégias de implementação da Década da Educação para o Desenvolvimento Sustentável.

Destaca-se que o Brasil ocupa a $9^{a}$ posição na Scopus e $11^{a}$ posição na WOS, no ranking das publicações relacionadas ao tema pesquisado, o que corrobora com o fato de que no Brasil, a relação entre Educação e Sustentabilidade está presente como exigência e determinação desde a Constituição Federal Brasileira - CFB de 1988 (CFB, 1988, p. 127-28).

\subsection{Educação e Sustentabilidade - os "hot topics"}

Nesta etapa da pesquisa, foram investigados os principais tópicos relacionados à educação para sustentabilidade que estão sendo estudados nos últimos anos. Com base em 
uma análise prévia das publicações encontradas na WOS, foram selecionados 20 tópicos relacionados à temática.

O Quadro 10 classifica os referidos tópicos relacionados à temática Educação e Sustentabilidade, selecionados conforme o número de publicações:

\begin{tabular}{|l|c|}
\hline \multicolumn{1}{|c|}{ Área temátic } & No de publicações $^{\mathbf{0}}$ \\
\hline Gestão (Management) & 1.137 .180 \\
\hline Saúde (Health) & 962.790 \\
\hline Meio ambiente (Environment) & 929.844 \\
\hline Estratégia (Strategy) & 873.728 \\
\hline Tecnologia (Technology) & 798.471 \\
\hline Social (Social) & 647.403 \\
\hline Aprendizagem (Learning) & 467.976 \\
\hline Planejamento (Planning) & 436.127 \\
\hline Engenharia (Engineering) & 392.128 \\
\hline Administração pública (Government) & 170.837 \\
\hline Economia (Economy) & 161.016 \\
\hline Saúde pública (Public Health) & 132.725 \\
\hline Inovação (Innovation) & 102.111 \\
\hline Estudos urbanos (Urban Studies) & 78.322 \\
\hline Agricultura (Agriculture) & 74.797 \\
\hline Ciências Sociais (Social Sciences) & 52.933 \\
\hline Recursos Hídricos (Water Resources) & 47.011 \\
\hline Recursos Humanos (Human Resources) & 46.894 \\
\hline Governança (Governance) & 37.857 \\
\hline Responsabilidade social (Social Responsibility) & 15.063 \\
\hline
\end{tabular}

Quadro 10 Tópicos relacionados aos temas Education and Sustainability.

Fonte: $\operatorname{WOS}(\mathrm{Dez} / 2012)$.

Posteriormente, foi realizada a combinação de cada tópico listado no Quadro $11 \mathrm{com}$ a expressão Education and Sustainability, sendo calculado o total de publicações para cada combinação (tópico relacionado), o h-b e o coeficiente m.

\begin{tabular}{|l|c|c|c|}
\hline \multicolumn{1}{|c|}{ Tópicos - Base WOS } & $\begin{array}{c}\text { Total de } \\
\text { publicações }\end{array}$ & Índice h-b & Coeficiente $\boldsymbol{m}$ \\
\hline Saúde (Health) & 562 & 31 & 1,41 \\
\hline Gestão (Management) & 607 & 28 & 1,27 \\
\hline Meio ambiente (Environment) & 527 & 25 & 1,14 \\
\hline Estratégia (Strategy) & 428 & 24 & 1,09 \\
\hline Social (Social) & 595 & 23 & 1,05 \\
\hline Saúde pública (Public Health) & 151 & 22 & 1,00 \\
\hline Planejamento (Planning) & 308 & 20 & 0,91 \\
\hline Administração pública(Government) & 250 & 19 & 0,86 \\
\hline Aprendizagem (Learning) & 567 & 18 & 0,82 \\
\hline Agricultura (Agriculture) & 163 & 17 & 0,77 \\
\hline Tecnologia (Technology) & 401 & 17 & 0,77 \\
\hline
\end{tabular}




\begin{tabular}{|l|c|c|c|}
\hline Inovação (Innovation) & 163 & 13 & 0,59 \\
\hline Engenharia (Engineering) & 313 & 12 & 0,55 \\
\hline Recursos Hídricos (Water Resources) & 76 & 12 & 0,55 \\
\hline Recursos Humanos (Human Resources) & 117 & 12 & 0,55 \\
\hline Ciências Sociais (Social Sciences) & 127 & 11 & 0,50 \\
\hline Governança (Governance) & 64 & 10 & 0,45 \\
\hline Estudos urbanos (Urban Studies) & 68 & 9 & 0,41 \\
\hline Economia (Economy) & 148 & 8 & 0,36 \\
\hline Responsabilidade social (Social Responsibility) & 62 & 6 & 0,27 \\
\hline
\end{tabular}

Quadro 11 Hot topics no estudo sobre o tema pesquisado.

Fonte: WOS (Dez/2012).

Orientando-se pelas considerações de Banks (2006) não é possível classificar como hot topcis ou tópicos quentes as combinações dos temas Education and Sustainability, pois nenhum deles apresentou o coeficiente $\mathrm{m} \geq 2$.

Os tópicos Health (Saúde), Management (Gestão), Enviromental (Meio Ambiente), Strategy (Estratégia), Social (Social), Public Health (Saúde Pública), obtiveram um coeficiente m entre 0,5 e 2, são considerados como hot topics emergentes em suas áreas de pesquisa. Já os tópicos que apresentaram $\mathrm{m} \leq 0,5$ podem ser de interesse para pesquisadores em um campo específico de pesquisa.

Nesse sentido, denota-se que a temática Educação e Sustentabilidade consiste em um tema emergente, apresentando grandes desafios no que se refere ao desenvolvimento de seus estudos.

\subsection{Relação dos trabalhos com maior número de citações}

Nesta fase foram selecionadas dez (10) publicações, que obtiveram o maior número de citações em cada base, conforme está apresentado nos Quadros 12 e 13.

\begin{tabular}{|c|c|c|}
\hline $\mathbf{N}^{\mathbf{o}}$ & Titulo / Autor / Periódico / Ano & $\begin{array}{l}N^{0} \text { Citações } \\
1991 \text { a } 2012\end{array}$ \\
\hline 01 & $\begin{array}{l}\text { Title: Soil-transmitted helminth infections: ascariasis, trichuriasis, and } \\
\text { hookworm } \\
\text { Author(s): Bethony, J; Brooker, S; Albonico, M; et al. } \\
\text { Source: LANCET Volume: } 367 \text { Published: May } 62006\end{array}$ & 343 \\
\hline 02 & $\begin{array}{l}\text { Title: Key issues and research priorities in landscape ecology: An } \\
\text { idiosyncratic synthesis } \\
\text { Author(s): Wu, JG; Hobbs, } R \\
\text { Source: LANDSCAPE ECOLOGY Volume: } 17 \text { Published: } 2002\end{array}$ & 255 \\
\hline 03 & $\begin{array}{l}\text { Title: Planning for the sustainability of community-based health programs: } \\
\text { conceptual frameworks and future directions for research, practice and policy } \\
\text { Author(s): Shediac-Rizkallah, MC; Bone, LR } \\
\text { Source: HEALTH EDUCATION RESEARCH Volume: } 13 \text { Published: Mar }\end{array}$ & 151 \\
\hline
\end{tabular}




\section{UM PANORAMA SOBRE OS ESTUDOS RELACIONADOS À TEMÁTICA EDUCAÇÃO E SUSTENTABILIDADE \\ DOI: http://dx.doi.org/10.5007/1983-4535.2013v6n2p160}

\begin{tabular}{|c|c|c|}
\hline & 1998 & \\
\hline 04 & $\begin{array}{l}\text { Title: Sustaining Interventions In Community Systems - On The Relationship } \\
\text { Between Researchers And Communities } \\
\text { Author(s): Altman, DG } \\
\text { Source: HEALTH PSYCHOLOGY Volume: } 14 \text { Published: Nov } 1995\end{array}$ & 147 \\
\hline 05 & $\begin{array}{l}\text { Title: Meeting ecological and societal needs for freshwater } \\
\text { Author(s): Baron, JS; Poff, NL; Angermeier, PL; et al. } \\
\text { Source: ECOLOGICAL APPLICATIONS Volume: } 12 \text { Published: Oct } 2002\end{array}$ & 137 \\
\hline 06 & $\begin{array}{l}\text { Title: A randomized, controlled effectiveness trial of an AIDS prevention } \\
\text { program for low-income African-American youths } \\
\text { Author(s): Stanton, BF; Li, XM; Ricardo, I; et al. } \\
\text { Source: Archives of Pediatrics \& Adolescent Medicine Vol: } 150 \text { Publ: Apr } \\
1996\end{array}$ & 130 \\
\hline 07 & $\begin{array}{l}\text { Title: New Moves: a school-based obesity prevention program for adolescent } \\
\text { girls } \\
\text { Author(s): Neumark-Sztainer, D; Story, M; Hannan, PJ; et al. } \\
\text { Source: PREVENTIVE MEDICINE Volume: } 37 \text { Published: Jul } 2003\end{array}$ & 126 \\
\hline 08 & $\begin{array}{l}\text { Title: From safe yield to sustainable development of water resources - the } \\
\text { Kansas experience } \\
\text { Author(s): Sophocleous, M } \\
\text { Source: JOURNAL OF HYDROLOGY Volume: } 235 \text { Published: Aug } 222000\end{array}$ & 108 \\
\hline 09 & $\begin{array}{l}\text { Title: Local communities and protected areas: Attitudes of rural residents } \\
\text { towards conservation and machalilla national park, Ecuador } \\
\text { Author(s): Fiallo, EA, Jacobson, SK } \\
\text { Source: ENVIRONMENTAL CONSERVATION Volume: } 22 \text { Published: } 1995\end{array}$ & 103 \\
\hline 10 & $\begin{array}{l}\text { Title: Effectiveness of alliances and partnerships for health promotion } \\
\text { Author(s): Gillies, P } \\
\text { Source: HEALTH PROMOTION INTERNATIONAL Volume: } 13 \text { Publ: Jun } \\
1998\end{array}$ & 97 \\
\hline
\end{tabular}

Quadro 12 Principais artigos em números de citações.

Fonte: WOS (Dez/2012).

\begin{tabular}{|c|c|c|}
\hline $\mathbf{N}^{\mathbf{o}}$ & Titulo / Autor / Periódico / Ano & $\begin{array}{l}\mathrm{N}^{0} \text { Citações } \\
1991 \text { a } 2012\end{array}$ \\
\hline 01 & $\begin{array}{l}\text { Title: Soil-transmitted helminth infections: ascariasis, trichuriasis, and } \\
\text { hookworm } \\
\text { Author(s): Bethony, J; Brooker, S; Albonico, M; et al. } \\
\text { Source: LANCET Volume: } 367 \text { Published: May } 62006\end{array}$ & 376 \\
\hline 02 & $\begin{array}{l}\text { Title: Key issues and research priorities in landscape ecology: An } \\
\text { idiosyncratic synthesis } \\
\text { Author(s): Wu, JG; Hobbs, } R \\
\text { Source: LANDSCAPE ECOLOGY Volume: } 17 \text { Published: } 2002\end{array}$ & 277 \\
\hline 03 & $\begin{array}{l}\text { Title: Enhancing School-Based Prevention and Youth Development Through } \\
\text { Coordinated Social, Emotional, and Academic Learning } \\
\text { Author(s): Greenberg, M.T., Weissberg, R.P., O'Brien, M.U., Zins, J.E., } \\
\text { Fredericks, L., Resnik, H., Elias, M.J., } \\
\text { Source: AMERICAN PSYCHOLOGIST Volume: } 58 \text { Published: } 2003\end{array}$ & 239 \\
\hline 04 & $\begin{array}{l}\text { Title: Participatory learning for sustainable agriculture } \\
\text { Author(s): Pretty, J.N., } \\
\text { Source: WORLD DEVELOPMENT Volume: } 23 \text { Published: } 1995\end{array}$ & 219 \\
\hline 05 & Title: Issues in disseminating and replicating effective prevention programs & 202 \\
\hline
\end{tabular}




\begin{tabular}{|c|c|c|}
\hline $\mathbf{N}^{0}$ & Titulo / Autor / Periódico / Ano & $\begin{array}{l}N^{0} \text { Citações } \\
1991 \text { a } 2012\end{array}$ \\
\hline & $\begin{array}{l}\text { Author(s): Elliott, D.S., Mihalic, S., } \\
\text { Source: PREVENTION SCIENCE Volume: } 5 \text { Published: } 2004\end{array}$ & \\
\hline 06 & $\begin{array}{l}\text { Title: Rapid diagnostic tests compared with malaria microscopy for guiding } \\
\text { outpatient treatment of febrile illness in Tanzania: Randomised trial } \\
\text { Author(s): Reyburn, H., Mbakilwa, H., Mwangi, R., Mwerinde, O., Olomi, R., } \\
\text { Drakeley, C., Whitty, C.J.M., } \\
\text { Source: BRITISH MEDICAL JOURNAL Vol: } 334 \text { Published: } 2007\end{array}$ & 153 \\
\hline 07 & $\begin{array}{l}\text { Title: Meeting ecological and societal needs for freshwater } \\
\text { Author(s): Baron, J.S., LeRoy Poff, N., Angermeier, P.L., Dahm, C.N., Gleick, } \\
\text { P.H., Hairston Jr., N.G., Jackson, R.B., Johnston, C.A., Richter, B.D., } \\
\text { Steinman, A.D., } \\
\text { Source: ECOLOGICAL APPLICATIONS Vol: } 12 \text { Published: } 2002\end{array}$ & 153 \\
\hline 08 & $\begin{array}{l}\text { Title: New Moves: a school-based obesity prevention program for adolescent } \\
\text { girls } \\
\text { Author(s): Neumark-Sztainer, D., Story, M., Hannan, P.J., Rex, J., } \\
\text { Source: PREVENTIVE MEDICINE Volume: } 37 \text { Published: Jul } 2003\end{array}$ & 135 \\
\hline 09 & $\begin{array}{l}\text { Title: Hunger, waiting time and transport costs: Time to confront challenges to } \\
\text { ART adherence in Africa } \\
\text { Author(s): Hardon, A.P., Akurut, D., Comoro, C., Ekezie, C., Irunde, H.F., } \\
\text { Gerrits, T., Kglatwane, J., Kinsman, J., Kwasa, R., Maridadi, J., Moroka, T.M., } \\
\text { et. al. } \\
\text { Source: AIDS Care - Psychological and Socio-Medical Aspects of AIDS/HIV } \\
\text { Volume: } 19 \text { Published: } 2007\end{array}$ & 125 \\
\hline 10 & $\begin{array}{l}\text { Title: Family planning: the unfinished agenda } \\
\text { Author(s): Cleland, J., Bernstein, S., Ezeh, A., Faundes, A., Glasier, A., Innis, } \\
\text { J., } \\
\text { Source: LANCET Volume: } 368 \text { Publ: Jun } 2006\end{array}$ & 124 \\
\hline
\end{tabular}

Quadro 13 Principais artigos em números de citações.

Fonte: Scopus (Dez/2012).

A pesquisa referente aos trabalhos com maior número de citações teve como objetivo verificar a relação das publicações mais citadas com os autores que mais publicaram nesse mesmo período, apresentados no Quadro 6.

Percebe-se que em ambas as bases de dados, os autores das publicações mais citadas não estão entre os autores que mais publicaram no período. Ainda é possível constatar que as publicações com maior número de citações estão relacionadas à área da saúde, tendo em vista que a temática educação e sustentabilidade é de fundamental importância em questões relacionadas a este contexto. Destaca-se que os temas relacionados à saúde estão entre as áreas prioritárias definidas no Plano de implementação da Década da Educação das Nações Unidas para o Desenvolvimento Sustentável (UNESCO, 2005). 
Constatou-se ainda que, quatro publicações aparecem entre as mais citadas em ambas as bases de dados, as publicações que ocupam $1^{\circ}$ e $2^{\circ}$ lugar no ranking das mais citadas são as mesmas tanto na base WOS quanto na Scopus.

\section{CONSIDERAÇÕES FINAIS}

A realização deste estudo permitiu constatar que o tema educação para a sustentabilidade está em evolução. Comparando-se ambas as bases pesquisadas verificou-se que o número de publicações aumentou consideravelmente nos últimos 10 anos. Entre os periódicos com maior número de publicações na base WOS, destaca-se o Journal of Cleaner Production e na base Scopus a ASSE Annual Conference and Exposition Conference Proceedings.

Os Estados Unidos lidera o ranking dos países que mais publicam sobre a temática nas duas bases. Na WOS a Austrália é o segundo país que mais publica, seguido por Inglaterra, Canadá e Alemanha. Na Scopus, o Reino Unido é segundo que mais publica, seguido por Austrália, Canada e Alemanha. Destaca-se que o Brasil ocupa a $11^{\mathrm{a}}$ posição no ranking da WOS e $9^{\circ}$ na Scopus.

Os tópicos Health (saúde), Management (gestão), Enviromental (meio ambiente), obtiveram um coeficiente $\mathrm{m}$ entre 0,5 e 2 . Estes então podem ser considerados como "hot topics" emergentes em suas áreas de pesquisa. Na busca de um quadro comparativo entre as publicações mais citadas versus os autores que mais publicaram no período pesquisado, foi possível verificar que as publicações com o maior número de citações não pertencem aos autores que mais publicam sobre o mesmo tema.

No decorrer do trabalho, foi possível verificar a utilidade de mecanismos de busca como a WOS e a Scopus para a realização de pesquisas acadêmicas, que servem de ferramentas para que a comunidade acadêmica tenha acesso às publicações, bem como, buscar informações a respeito da evolução de seus temas de interesse.

Diante do estudo apresentado sobre a temática educação e sustentabilidade, verifica-se o crescimento de estudos relacionados a temática, visto pela sua importância para o campo do ensino, pesquisa, bem como para mobilização da sociedade em prol do desenvolvimento sustentável.

Como limitação do estudo, destaca-se a sua realização utilizando apenas duas bases de dados específicas. Por esta razão, sugere-se que estudos futuros desta natureza, possuam uma 
amplitude maior, abrangendo, por exemplo, eventos acadêmicos nacionais e internacionais, periódicos científicos ou outras importantes bases de dados científicos.

\section{REFERÊNCIAS}

BANKS, M. G. An extension of the Hirsch index: indexing scientific topics and compounds. 2006. Disponível em <http://www.arxiv.org/abs/physics/0604216 $>$ Acesso em Jul. 2010.

BARBIERI, J. C. Organizações inovadoras sustentáveis. In: BARBIERI, J. C.; SIMANTOB, M. A. (Org.). Organizações inovadoras sustentáveis: uma reflexão sobre o futuro das organizações. São Paulo: Atlas, 2007.

BAR-ILAN J. Which h-index? A comparison of WOS, Scopus and Google Scholar. Scientometrics, v. 74, n. 2, p. 257-271, 2008.

BRASIL. Constituição da República Federativa do Brasil: promulgada em 5 de outubro de 1988. Disponível em: <www.planalto.gov.br>. Acesso em: Dez. 2012.

BRASIL. Lei Federal $\mathbf{n}^{0}$ 9.795, de 27 de abril de 1999. Dispõe sobre a educação ambiental, institui a Política Nacional de Educação Ambiental e dá outras providências. Disponível em:

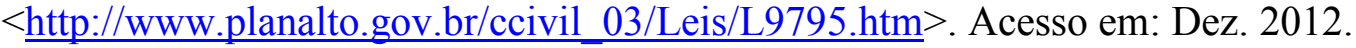

BRITO, E. P. Z.; LOMBARDI, M. S. Desenvolvimento sustentável como fator de competitividade. In: ENANPAD, 31, 2007, Rio de Janeiro. Anais. Rio de Janeiro: ANPAD, 2007.

CAPES - COORDENAÇÃO DE APERFEIÇOAMENTO DE PESSOAL DE NÍVEL SUPERIOR. Acervo. 2012. Disponível em: $<$ http://www.periodicos.capes.gov.br.ez47. periodicos.capes.gov.br/index.php?option $=\mathrm{com}$ pcollection $\& \mathrm{mn}=70 \& \mathrm{smn}=79>$. Acesso em: Dez. 2012.

COMISSÃO MUNDIAL SOBRE MEIO AMBIENTE E DESENVOLVIMENTO-CMMAD. Nosso futuro comum. Rio de Janeiro: Fundação Getúlio Vargas - FGV, 1991.

DIÁRIO OFICIAL DA UNIÃO - DOU. A responsabilidade social e sustentabilidade como disciplina. Resolução $\mathrm{n}^{\mathrm{o}}$ 2, 15 junho de 2012. Disponível em:

$<$ http://www.in.gov.br/imprensa/visualiza/index.jsp?jornal=1\&pagina $=71 \&$ data $=18 / 06 / 2012>$ . Acesso em: Out. 2012.

DIAS, R. Gestão ambiental: Responsabilidade social e sustentabilidade. São Paulo: Atlas, 2007.

ENGELMAN, R.; FRACASSO, E. M.; TOMETICH, P. Gestão ambiental em universidades brasileiras. Anais do XIV ENGEMA. São Paulo, 2012. 
GABRIEL, F. S.; MORIGUCHI, S. N. Avaliação do curso de administração da UFU: validando os 8 A's. In: VII Seminários em Administração - SEMEAD, 2005, São Paulo. Anais. São Paulo, 2005.

GADOTTI, M. Educar para a sustentabilidade: uma contribuição à década da educação. para o desenvolvimento sustentável. São Paulo: Editora e Livraria Instituto Paulo Freire, 2008 .

HIRSCH, J. E. An index to quantify an individual's scientific research output. Proceedings of the National Academy of Sciences of the United States of America, v. 102, n. 46, p. 16569-16572, 2005.

HOFF, D. N. A construção do desenvolvimento sustentável através das relações entre as organizações e seus stakeholders: a proposição de uma estrutura analítica. 2008. 425p. Tese (Doutorado em Agronegócios) - Universidade Federal do Rio Grande do Sul, Porto Alegre, 2008 .

JACOBI, P. Educação ambiental, cidadania e sustentabilidade. Cadernos de Pesquisa, $\mathrm{n}$. 118, p. 189-205, março/ 2003.

MACEDO, M. A. S.; CASA NOVA, S. P.; ALMEIDA, K. Mapeamento e análise bibliométrica da utilização da análise envoltória de dados (DEA) em estudos das áreas de contabilidade e administração. In: ENANPAD, 23, 1999, Foz do Iguaçu. Anais. Foz do Iguaçu: ANPAD, 1999.

MINISTÉRIO DA EDUCAÇÃO - ME. Sistema de acompanhamento de processos das instituições de ensino superior - SAPIEnS / Plano de Desenvolvimento Institucional / Diretrizes para elaboração. 2012. Disponível em: <http://www2.mec.gov.br/sapiens/pdi.htm>. Acesso em: Mai. 2012.

NOLET, V.; GILDA W. Education for Sustainability in Washington State: A Whole Systems Approach. The Journal of Sustainability Education. Disponível em $<$ http://www.jsedimensions.org/wordpress/content/education-for-sustainability-inwashington-state-a-whole-systems-approach 2010 05/>. Acesso em: Jan. 2012.

ORGANIZAÇÃO DAS NAÇÕES UNIDAS PARA A EDUCAÇÃO, A CIÊNCIA E A CULTURA - UNESCO. Década da Educação das Nações Unidas para um

Desenvolvimento Sustentável, 2005-2014: documento final do esquema internacional de implementação. Brasília: UNESCO, 2005.

ROSTAING, H. La bibliométrie et sés techniques. Toulouse: Sciences de la Société; Marseille: Centre de Recherche Rétrospective de Marseille, 1997.

ROWE, D. E. O. Perspectivas do ensino-aprendizagem e habilidades necessárias ao administrador: um estudo de caso. Revista ANGRAD. v. 5, n. 1, 2005. 
SILVA, M. R. Análise bibliométrica da produção científica docente do programa de pósgraduação em educação especial/UFSCar: 1998-2003. Dissertação (Mestrado em Educação Especial) - Universidade Federal de São Carlos, São Carlos, 2004.

STEPHENS, J. C.; HERNANDEZ, M. E.; ROMÁN, M.; GRAHAM, A. C.; SCHOLZ, R. W. (2008). Higher education as a change agent for sustainability in different cultures and contexts. International Journal of Sustainability in Higher Education, v.9, n.3, p.317-338, 2008.

TAMAIO, I. A mediação do professor na construção do conceito de natureza. Campinas, 2000. 254 p. Dissertação (Mestradro em Geociências). Faculdade de Educação - Unicamp, 2000 .

ZITZKE, V. A. Educação ambiental e eco desenvolvimento. Revista Eletrônica do Mestrado em Educação Ambiental, 9, p.175-188, jul.-dez. 2002. 